\title{
Synthesis of ibuprofen-functionalized hydrogels
}

\section{as an anti-inflammatory drug delivery system}

Emanuele Mauri ${ }^{\mathrm{a}, \mathrm{b}, \dagger}$, Arianna Rossetti ${ }^{\mathrm{b}, \dagger}$, Pamela Mozetic ${ }^{\mathrm{c}}$, Chiara Schiavon ${ }^{\mathrm{b}}$, Alessandro Sacchetti ${ }^{\mathrm{b}}$, Alberto Rainer ${ }^{a, *}$, Filippo Rossi ${ }^{b, *}$

${ }^{a}$ Department of Engineering, Tissue Engineering and Chemistry for Engineering Unit, Università Campus BioMedico di Roma, via Alvaro del Portillo 21, 00128 Rome, Italy

b Department of Chemistry, Materials and Chemical Engineering “Giulio Natta”, Politecnico di Milano, via L. Mancinelli 7, 20131 Milan, Italy

${ }^{c}$ Center for Translational Medicine, International Clinical Research Center, St. Anne's University Hospital, Pekařská 929/56, 60200 Brno, Czech Republic

$\dagger$ equally contributed

* Alberto Rainer. Tel.: +39 06225419214; fax: +39 06225419419; e-mail: a.rainer@unicampus.it

* Filippo Rossi. Tel.: +39 0223993145; fax: +39 0223993180; e-mail: filippo.rossi@polimi.it 


\begin{abstract}
Ibuprofen (IBU) is a non-steroidal anti-inflammatory drug (NSAID) commonly used in the treatment of pain, fever and inflammation. However, the administration of IBU in its free carboxylic acid form is strongly dependent on its limited solubility in aqueous solution. This mandates for an increased drug concentration to reach the therapeutic window, and promotes the alternative use of IBU sodium salt, even if this latter form poses significant constraints in terms of tunable release due to its uncontrolled and rapid diffusion. A potential solution is represented by oral administration through physical encapsulation of ibuprofen in designed carriers, despite this route limits the application of this therapeutic. In this work, we propose the covalent tethering of ibuprofen to a hydrogel matrix via esterification reaction. Exploiting the cleavability of the ester bond under physiological conditions, we propose a controlled drug delivery system where the whole drug payload can be released, thus overcoming the questioned aspects of over-dosage and solubility-dependent administration. In particular, we tested the biological activity of cleaved ibuprofen in terms of cyclooxygenase inhibition, reporting that chemical tethering did not alter the efficiency of the NSAID. Moreover, due to the solgel transition of the hydrogel matrix, these ibuprofen-functionalized hydrogels could be used as injectable tools in several clinical scenarios, performing a localized drug release and opening advanced avenues for in situ treatments.
\end{abstract}

Keywords: ibuprofen; hydrogel; ester hydrolysis; cyclooxygenase; controlled drug delivery 


\section{Introduction}

The clinical scenario regarding inflammatory response, mild-to-moderate pain, fever and post-op recovery is generally addressed using non-steroidal anti-inflammatory drugs (NSAID). Ibuprofen (IBU) represents a common NSAID thanks to its anti-inflammatory, analgesic and antipyretic properties[1]. Its mechanism of action regards the inhibition of the cyclooxygenase isoenzymes (COX) responsible for the conversion of arachidonic acid into prostaglandins, autacoids with a leading role in promoting pain, inflammation and fever. In particular, COX-2 is related to inflammation and activated immune response $[2,3]$. However, standard administration routes of ibuprofen are characterized by some constraints regarding the drug dosage and its solubility in physiological environment. In details, due to its first-pass metabolism and its limited absorption, ibuprofen is currently administered using a drug concentration 10 times higher than the required dosage for a therapeutic benefit, and the oral route is preferentially selected, through the use of capsules, tablets or dispersions[4, 5]. Moreover, the poor water-solubility and low dissolution rate of IBU reduce its biological availability at the target site, resulting in an accumulation in other districts which leads to severe adverse consequences, including gastrointestinal or kidney disorders: gastritis, dyspepsia, epigastric pain, peptic ulcer, hypertension, bronchospasm and heart burn[1, 6-8]. Different strategies have been proposed to overcome the critical issues of IBU solubility and guarantee a controlled drug delivery. Surfactants[9], block copolymers[10, 11] and dendrimers[12] have been added to the drug suspensions in order to enhance IBU hydrophilicity, through the reciprocal physical, polar and ionic interactions[13-15]. However, the resulting formulations appear sensitive to temperature and $\mathrm{pH}$ variations, setting a system of improved drug solubility essentially in physiological conditions[16]. Furthermore, the tuning of drug release requires a niche capable of drug protection and release under specific external stimuli[17, 18]. 
The design of polymeric scaffolds as drug carriers with specific physical and chemical features could represent an advanced approach to ensure IBU biodistribution and would overcome the rapid and unwanted in vivo clearance of the administered drug. As is generally known, hydrogels play a leading role in drug delivery: they are three-dimensional networks of chemically or physically cross-linked polymer chains, and as such, they are characterized by porous structures allowing the diffusion of metabolites, ions, nutrients, active principles and signaling molecules to and from the surrounding environment to maintain the biological balance[19, 20]. Moreover, the outward/inward diffusion of biomolecules in hydrogels occurs preserving the network physical structure[21]. The entrapment of IBU within a hydrogel matrix is a facile method to protect the drug and to perform its release in a confined area, but controlled drug diffusion mechanism, improved drug solubility and availability need to be addressed through chemical functionalization strategies[22-25]. The smart and reversible modification of the terminal carboxyl group of IBU and its subsequent tether to a polymeric scaffold represent a promising solution to overcome the critical issues limiting the versatile application of IBUbased treatment in a wide range of clinical scenarios. In this work, we propose the synthesis of IBUtethered agarose hydrogels via esterification strategy. In particular, to promote the formation of ester bonds between the drug and the hydrogel matrix, a validated formulation[26, 27] has been modified by the addition of 1,3-propanediol, that has been introduced in a microwave-assisted synthetic route to become an integral part of the scaffold design. At physiological conditions, ester is a cleavable bond and promotes release of the drug in its pristine form. The chemical functionalization of a therapeutic molecule is generally a questionable aspect: the partial modification of the molecular structure could affect its pharmacokinetics, purity, biocompatibility and efficacy, hampering its application. For these reasons, we evaluated the released IBU in terms of $\mathrm{COX}$ inhibition potential, reporting an antiinflammatory performance comparable to pristine IBU in aqueous medium, ruling out potential drawbacks related to IBU chemical modifications. 
Moreover, in virtue of its sol-gel transition and its elastic rather than viscous behavior, the hydrogel could be used as an injectable system. These remarks highlight the potential application of several inflammatory conditions including osteoarthritis, cartilage regeneration and melanoma, all clinical scenarios where IBU is generally used in a physically entrapped form in different configurations[2830]. Indeed, the discussed chemical functionalization strategy can ensure a sustained drug release, improving the benefits, avoiding under- and overdosing, and modulating the pharmacokinetics by tuning the cleavability of the ester linker, thus opening new prospects in pharmacological treatments. 


\section{Material and Methods}

\subsection{Reagents and analysis techniques}

The hydrogel polymeric scaffold was synthetized using branched polyacrylic acid (Carbomer 974P, MW = 1 MDa, Fagron UK Ltd, UK), 1-3 propanediol (Merk KGaA, Darmstadt, Germany) and ultrapure agarose (DNA Grade, Mw 200,000 Da, density $1.64 \mathrm{~g} \mathrm{~cm}^{-3}$ ) from VWR international ltd (UK). Gelling and melting temperature ranges of this type of agarose are $34-37{ }^{\circ} \mathrm{C}$ and $60-90{ }^{\circ} \mathrm{C}$ respectively. Ibuprofen $((S)-(+) 2-(4-i s o b u t y l p h e n y l)$ propionic acid) was purchased from TCI America (USA), whereas (S)-ibuprofen sodium salt analytical standard was obtained from Merck KGaA. All other chemicals and solvents were purchased from Merck KGaA and were of analytical grade. Ibuprofen derivative was stored at $4{ }^{\circ} \mathrm{C}$. The NMR experiments were carried out on a Bruker AC (400 $\mathrm{MHz})$ spectrometer using chloroform $\left(\mathrm{CDCl}_{3}\right)$ or deuterium oxide $\left(\mathrm{D}_{2} \mathrm{O}\right)$ as solvents, and chemical shifts were reported as $\delta$ values in parts per million with respect to the tetramethylsilane solvent (TMS) as internal standard. FT-IR analysis were conducted using a Nicolet Nexus 6700 spectrometer coupled to a Nicolet Continuum Infrared microscope (Thermo Fisher Scientific) equipped with a 15× Reflachromat Cassegrain objective, at room temperature in air, setting a wavenumber range of 4000$500 \mathrm{~cm}^{-1}$ with 64 accumulated scans and at a resolution of $4 \mathrm{~cm}^{-1}$. The analyzed samples were prepared using the $\mathrm{KBr}$ pellet technique. Electrospray ionization mass spectrometry (ESI-MS) analyses were conducted on an Esquire 3000 Plus spectrometer, using methanol as a solvent.

\subsection{Synthesis of IBU-diol derivative}

Ibuprofen (2.8 g, 1 eq.) was dissolved in DCM at a concentration of $0.2 \mathrm{M}$ in a flask equipped with a dripper and maintained under $\mathrm{N}_{2}$ atmosphere. 
The flask was then cooled to $0{ }^{\circ} \mathrm{C}$ in an ice bath, and 4-(dimethylamino)pyridine (DMAP, $58.04 \mathrm{mg}$, 0.035 eq.) and 1,3-propanediol (1.14 mL, 1.15 eq.) were added to the solution. Successively, $\mathrm{N}_{,} \mathrm{N}^{\prime}-$ dicyclohexylcarbodiimide (DCC, 2.25 g, 0.8 eq.) was dissolved in DCM and added to the system dropwise. The resulting mixture was then brought to room temperature and kept stirring for $24 \mathrm{~h}$ under $\mathrm{N}_{2}$ atmosphere. The resulting system was filtered through a Büchner funnel, in order to remove the solid by-product (dicyclohexylurea) precipitated during the reaction. Following vacuum evaporation, the crude product was recovered through extraction with ethyl acetate and sequentially washed with saturated aq. $\mathrm{Na}_{2} \mathrm{CO}_{3}$ and $0.1 \mathrm{M} \mathrm{HCl}$. The recovered phase was dried over anhydrous sodium sulphate and the ethyl acetate was evaporated under vacuum. The obtained crude product was further purified on a silica gel column using a 8:2 hexane/ethyl acetate mixture (yield: $83 \%$ ).

${ }^{1} \mathrm{H}-\mathrm{NMR}(400 \mathrm{MHz}$, Chloroform- $d$ ) $\delta 7.19(\mathrm{~d}, J=8.1 \mathrm{~Hz}, 2 \mathrm{H}), 7.09$ (d, $J=8.1 \mathrm{~Hz}, 2 \mathrm{H}), 4.28-4.16$ (m, 2H), $3.70(\mathrm{q}, J=7.2 \mathrm{~Hz}, 1 \mathrm{H}), 3.54(\mathrm{t}, J=6.2 \mathrm{~Hz}, 2 \mathrm{H}), 2.45(\mathrm{~d}, J=7.1 \mathrm{~Hz}, 2 \mathrm{H}), 1.91-1.75(\mathrm{~m}$, 3H), 1.57 (s, 1H), 1.49 (d, $J=7.2 \mathrm{~Hz}, 3 \mathrm{H}), 0.89$ (d, $J=6.6 \mathrm{~Hz}, 6 \mathrm{H})$.

MS (ESI): $\mathrm{m} / \mathrm{z}$ calcd for $\mathrm{C}_{16} \mathrm{H}_{24} \mathrm{O}_{3}: 264,17[\mathrm{M}]^{+}$; found: $287.10[\mathrm{M}-\mathrm{Na}]^{+}$

\subsection{Ibuprofen-hydrogel synthesis}

Hydrogels with covalent IBU functionalization (cIBU-HG) were synthetized as follows: carbomer 974P (50 mg) was dissolved in phosphate-buffered saline (PBS) solution $(9.95 \mathrm{~mL})$ and the system left rest for $1 \mathrm{~h}$. Then, the $\mathrm{pH}$ was adjusted to 7.8 using $1 \mathrm{M} \mathrm{NaOH}$. The IBU-diol derivative was added a final concentration of $1 \mathrm{mg} / \mathrm{mL}$. The mixture $(5 \mathrm{~mL})$ was blended with agarose $(0.5 \% \mathrm{w} / \mathrm{v})$ and the system was subjected to microwave irradiation $(500 \mathrm{~W})$ for $30 \mathrm{~s}$, heating up to $80{ }^{\circ} \mathrm{C}$ to induce the condensation reaction between the carboxyl and hydroxyl groups. 
The reactor was kept closed to minimize evaporation, and upon cooling to $55{ }^{\circ} \mathrm{C}$, it was poured in steel cylindrical molds (11 mm diameter, $250 \mu \mathrm{L}$ each), where gelation was completed. Reference hydrogels (ref-HG) to be used as a control for this work were synthetized following the same procedure of cIBU-HG without the addition of modified propanediol, but adding a corresponding amount of IBU sodium salt following microwave initiation. Ref-HG hydrogels $(250 \mu \mathrm{L})$ were cast as previously described.

\subsection{Rheological characterization}

Rheological properties of cIBU-HG and ref-HG samples were evaluated using a MCR302 rheometer (Anton Paar $\mathrm{GmbH}$, Austria) in a $25 \mathrm{~mm}$ plate-plate configuration. The oscillatory responses $\left(\mathrm{G}^{\prime}\right.$, elastic modulus and $\mathrm{G}^{\prime \prime}$, loss/viscous modulus) were determined at low values of strain $(\gamma=1 \%)$ over the frequency range $0.01-20 \mathrm{~Hz}$. The pseudoplastic behavior was also investigated. The behaviors of shear stress and viscosity as a function of shear rate were examined and the linearity of the viscoelastic properties was checked (Supplementary data). The determination of the gelation point was conducted setting the rheometer's plate to $55^{\circ} \mathrm{C}$, to avoid premature gelation that could affect the results, and then applying a cooling curve until $20^{\circ} \mathrm{C}$, at constant strain $\gamma=1 \%$ and frequency $1 \mathrm{~Hz}$. These parameters were chosen referring to the $\mathrm{G}^{\prime}$ and $\mathrm{G}^{\prime \prime}$ values that were constant and independent in the frequency

range $0.01-20 \mathrm{~Hz}$ (frequency sweep) and in shear strain close to $1 \%$ (amplitude sweep). The temperature was set up to give some data for the polymeric solution at $55^{\circ} \mathrm{C}(1 \mathrm{~min})$ then get down to $20^{\circ} \mathrm{C}$ obtaining measurements for $1 \mathrm{~min}$, until the storage modulus becomes equal to the loss modulus, signifying the gelation point[31]. 


\subsection{Drug release}

The release of IBU from cIBU-HG and ref-HG specimens was evaluated at different $\mathrm{pH}$ values: 4.5, 7.4 and 8.5. Each hydrogel was submerged in $1 \mathrm{~mL}$ of aqueous solution at given $\mathrm{pH}$ and incubated at $37^{\circ} \mathrm{C}$. At defined time intervals, $300 \mu \mathrm{L}$ of eluate were withdrawn and spotted into 96-well plates (100 $\mu \mathrm{L}$ per well) for UV-vis spectrophotometric analysis. At each withdrawal, elution buffer was replenished with fresh medium to avoid mass transfer equilibrium between the hydrogel and the surrounding environment. Cumulative release from hydrogels was measured by UV-vis spectroscopy $(\lambda=264 \mathrm{~nm})$ on the basis of IBU standard calibration curves at the different $\mathrm{pH}$ conditions (Supplementary data). Data are presented as mean \pm standard deviation of three independent replicates.

\subsection{Cyclooxygenase (COX) Activity Assay}

The anti-inflammatory activity of IBU released from cIBU-HG was tested in vitro in terms of COX inhibition on cell lysates. The THP-1 monocytic cell line (American Tissue Culture Collection, ATCC; Rockville, MD) was used as a cell model. Cells were maintained in RPMI 1640 (Merck KGaA) supplemented with 10\% fetal bovine serum (FBS), 1\% non-essential amino acids, 1\% sodium pyruvate and $0.1 \mathrm{mg} / \mathrm{ml}$ penicillin/streptomycin/L-glutamine (Merck $\mathrm{KGaA}$ ). Cell differentiation into macrophages was performed in $75 \mathrm{~cm}^{2}$ flasks at a density of 1 x $10^{6}$ cells $/ \mathrm{mL}$ in complete RPMI containing $150 \mathrm{nM}$ phorbol 12-myristate 13-acetate (PMA) (Merck KGaA). Medium was changed after $24 \mathrm{~h}$ of treatment, and cells were cultured for additional $48 \mathrm{~h}$ in complete RPMI. Inflammation was induced by administration of $10 \mathrm{ng} / \mathrm{mL}$ LPS (Merck KGaA) for $48 \mathrm{~h}$. Cells were then washed with PBS and detached by trypsin. THP-1 macrophages were pelleted by centrifugation at $200 \mathrm{x} \mathrm{g}$ for 5 min and washed with PBS. 
The pellet was lysed in a lysis buffer consisting of ice-cold PBS, 1\% NP-40 surfactant (Thermo Fisher Scientific) and Halt Protease Inhibitor Cocktail (Thermo Fisher Scientific) and incubated on ice for 5 min. The lysate was centrifuged at $13,000 \mathrm{x}$ g for $5 \mathrm{~min}$ at $4{ }^{\circ} \mathrm{C}$ and the supernatant was collected. The COX inhibition effect of IBU was estimated using a Fluorometric Cyclooxygenase COX Activity Assay (Abcam, ab204699) according to the manufacturer's protocol, that provides a simple and sensitive method to detect the peroxidase activity of COX in biological samples. Eluates from cIBUHG were obtained as previously described following 24 incubation, and IBU concentration was determined spectrophotometrically. A freshly prepared IBU sodium salt at the same concentration was used as a positive control. Additionally, a no-IBU group was used for data normalization. In a typical experiment, $20 \mu \mathrm{L}$ of reaction buffer containing cell lysate, fluorometric probes and sample under investigation were spotted on a 96 well multiplate. After the addition of arachidonic acid (COX substrate) in $\mathrm{NaOH}$ solution, the enzymatic reaction led to the production of a fluorescent molecule (resorufin dye, $\lambda_{\mathrm{Ex} / \mathrm{Em}}=535 / 587 \mathrm{~nm}$ ), that could be monitored in kinetic mode ( 4 reads per min) for 20 min at room temperature (TECAN M200 Pro plate reader). Data were plotted as Relative Fluorescence Units (RFU) over time and COX activity could be determined as the slope of the curve in its linear region, using a resorufin standard calibration curve: in particular $1 \mathrm{COX}$ unit is defined as the amount that leads to the production of $1 \mu \mathrm{mol}$ of resorufin per min, at physiological $\mathrm{pH}$ and $25^{\circ} \mathrm{C}$.

\subsection{Statistical analysis}

Where applicable, experimental data were analyzed using Analysis of Variance (ANOVA) followed by post hoc testing. Statistical significance was at the 0.05 level. Results are presented as mean value \pm standard deviation. 


\section{Results and discussion}

\subsection{Material characterization}

The esterification between IBU and the diol represents an approach to modulate the mechanism of drug release exploiting the susceptibility to hydrolysis in biological environment: the ester linker is cleaved by hydrolytic reactions catalyzed by acids[32], bases[33], as well as proteins and enzymes such as albumins[34] and esterases[35, 36]. The corresponding kinetics is strictly dependent on the driving force able to disrupt the covalent bond[37, 38]. The reaction (Steglich esterification) between the IBU carboxyl group and the diol hydroxyl moiety meets the requirement of a chemoselective tool for controlled drug delivery. Our strategy is based on the smart functionalization of a single terminal -OH group of the propanediol (Figure 1), keeping the other available for the microwave-assisted polycondensation that leads to hydrogel formation. As such, IBU is covalently grafted to the polymeric scaffold, preventing its uncontrolled diffusion.
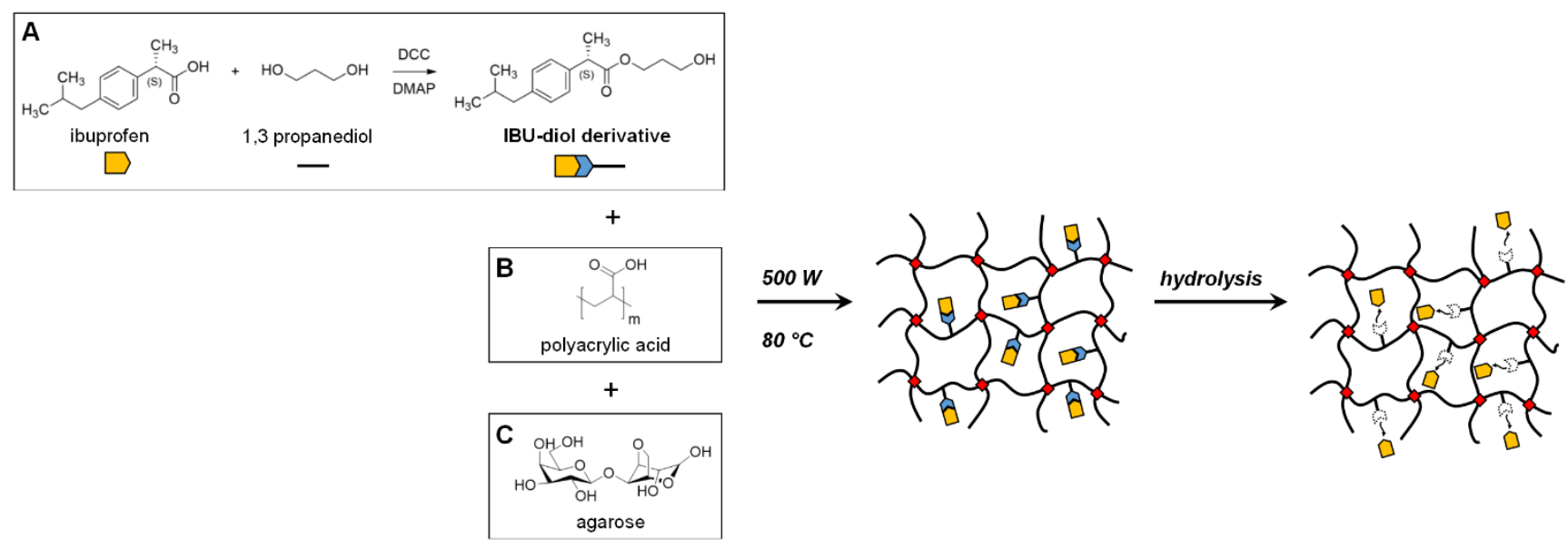

Figure 1. Scheme of functionalized hydrogels: A) Steglich esterification of IBU with 1,3-propanediol; B) carbomer 974P; C) agarose. 
The IBU-diol derivative was characterized by ${ }^{1} \mathrm{H}-\mathrm{NMR}$ analysis and ESI-mass analyses, that also confirmed the purity of the desired product (Figure 2).

A

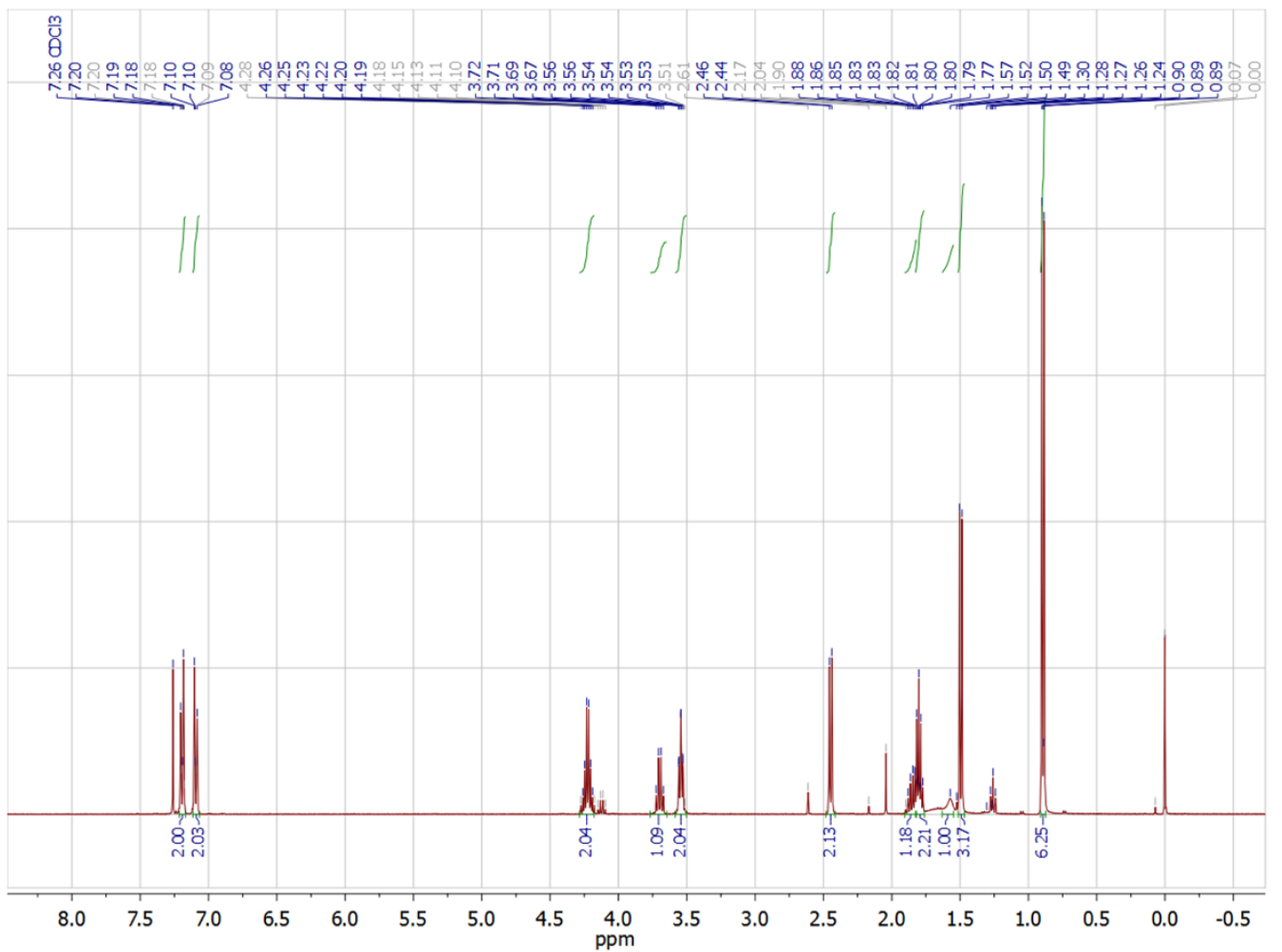

B

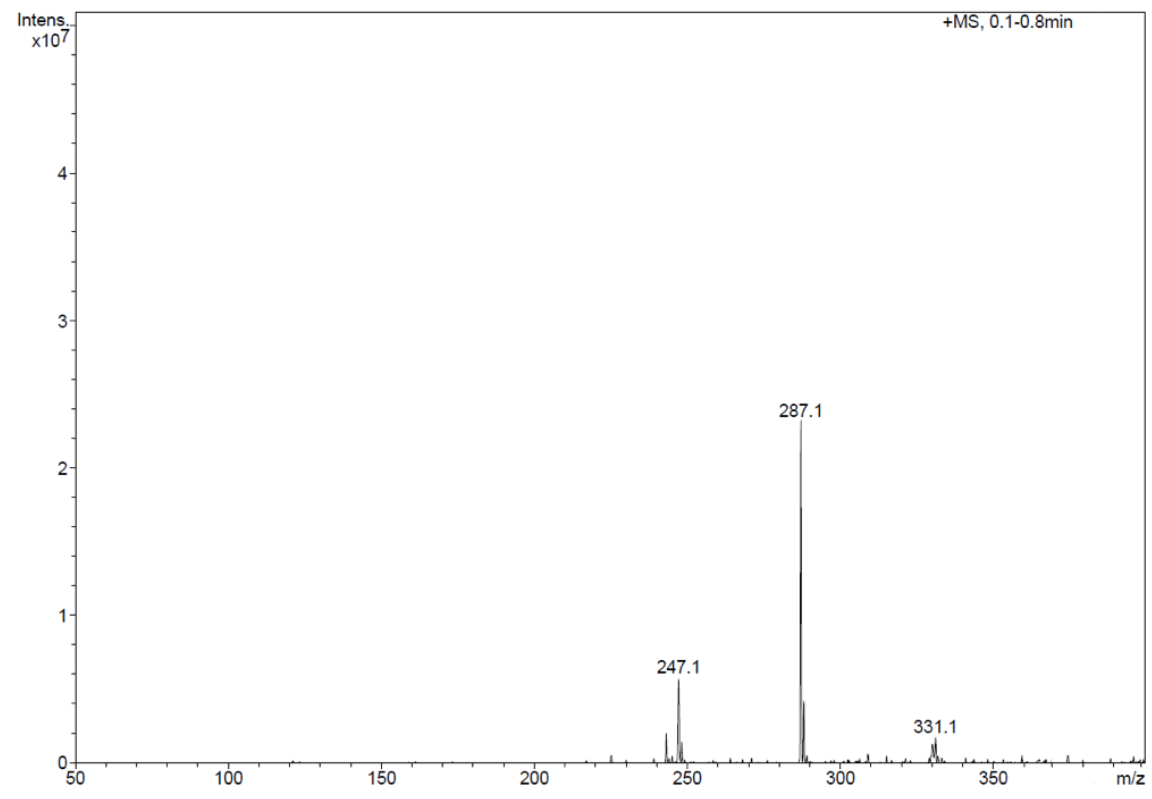

Figure 2. ${ }^{1} \mathrm{H}-\mathrm{NMR}$ spectrum (A) and ESI MS analysis (B) of IBU-diol derivative. 
${ }^{1} \mathrm{H}-\mathrm{NMR}$ spectrum shows a signal at $4.24 \mathrm{ppm}$ (multiplet) belonging to the methylene close to the ester group $\left(\mathrm{CH}_{2}\right.$-OOC) and a peak at $3.55 \mathrm{ppm}$ (triplet) related to the other tail of the propanediol component $\left(-\mathrm{CH}_{2}-\mathrm{OH}\right)$. The multiplet at $1.81 \mathrm{ppm}$ detects the methylene between the previous ones ($\left.\mathrm{CH}_{2}-\mathrm{CH}_{2}-\mathrm{OH}\right)$. The rest of the spectrum belongs to the IBU protons, confirming the diol monofunctionalization with the drug.

FT-IR spectra show the characteristic signals of diol-modified IBU, compared to pristine IBU (Figure 3). In the latter, the peak at $2950 \mathrm{~cm}^{-1}$ can be ascribed to IBU methyl asymmetric stretching, whereas the signals around $2869 \mathrm{~cm}^{-1}$ are related to the $-\mathrm{CH}_{2}$ asymmetric and symmetric stretching vibrations. The clear detectable signal at $1705 \mathrm{~cm}^{-1}$ is the stretch of $\mathrm{C}=\mathrm{O}$ in IBU and the $\mathrm{C}-\mathrm{C}$ stretching is visible at $1231 \mathrm{~cm}^{-1}$; all other signals in the wavenumber range $1100-500 \mathrm{~cm}^{-1}$ are related to the aromatic ring vibrations, in particular to $\mathrm{C}-\mathrm{H}$ and $=\mathrm{C}-\mathrm{H}$ in-plane deformation and to $\mathrm{C}=\mathrm{C}$ and $\mathrm{C}-\mathrm{C}$ in-plane ring deformation and bending[39]. The ester bond between the drug and 1,3-propanediol is recognizable at $1731 \mathrm{~cm}^{-1}$ as the stretching vibration of the resulting carbonyl group (the $\mathrm{C}=\mathrm{O}$ peak of pristine IBU remains visible as a shoulder signal) and at $1162 \mathrm{~cm}^{-1}$, where the asymmetric stretching of $\mathrm{C}-\mathrm{C}(=\mathrm{O})-\mathrm{O}$ occurs. Moreover, the vibrations ascribed to the carbon-oxygen stretching of the diol $\mathrm{O}-\mathrm{C}-\mathrm{C}$ at its esterified terminal is detectable at $1051 \mathrm{~cm}^{-1}$ and, finally, the presence of propanediol and its monofunctionalization is confirmed by the broad signal around $3350 \mathrm{~cm}^{-1}$ related to the stretching of terminal -OH groups. 


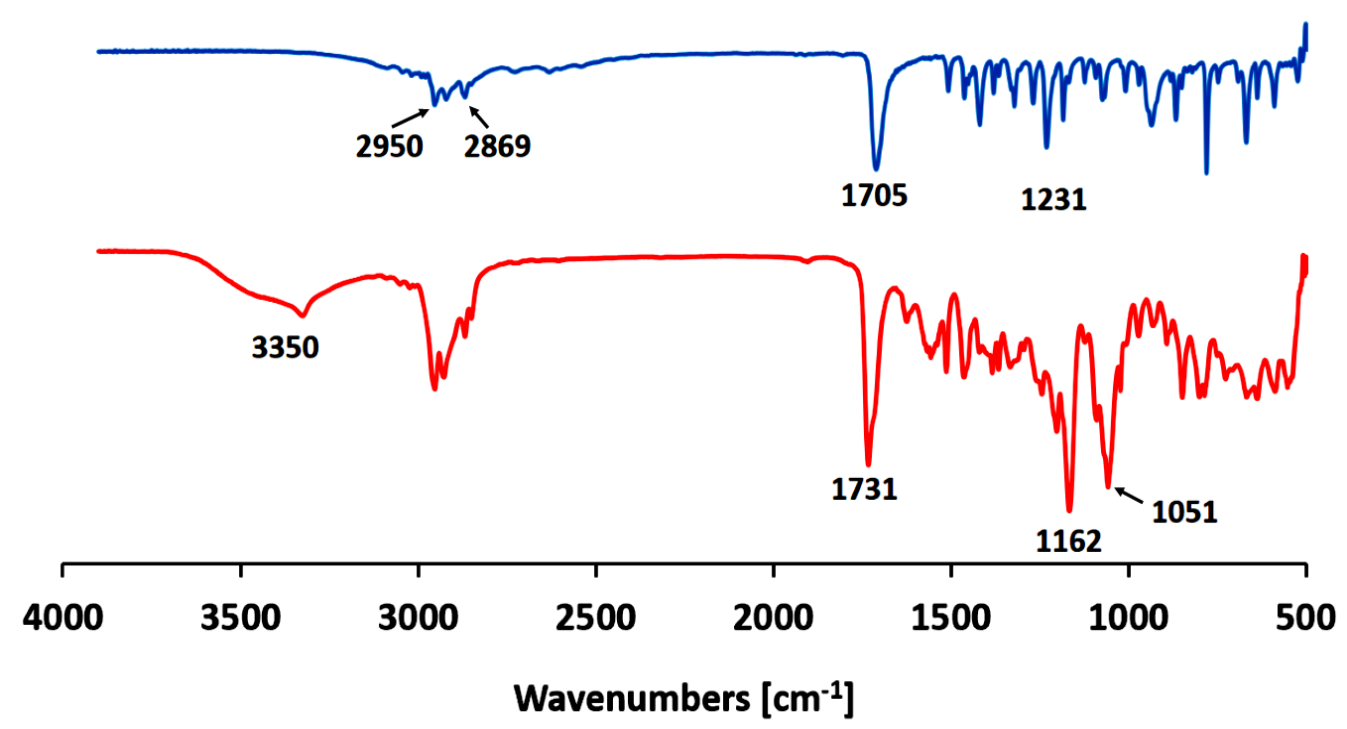

Figure 3. FT-IR spectra of ibuprofen (blue) and functionalized ibuprofen-propanediol (red). The characteristic signals are highlighted.

The hydrogel formation occurred through the chemical cross-linking among carbomer, agarose and the modified diol. In details, the 3D scaffold is mainly formed by the polyacrylic acid and agarose chains which, due to the higher macromer mobility at $80^{\circ} \mathrm{C}$, can enhance short-range interconnections among their functional groups. As polycondensation proceeds, local networks are produced (microgels) until the welding between their surfaces gives rise to the final three-dimensional macrostructure of cIBUHG. The in situ gelation properties of cIBU-HG were investigated, and compared to reference hydrogels (ref-HG). The sol-gel transition was monitored using the inverted tube test, that confirmed gelation within $7 \mathrm{~min}$ for both samples (Supplementary data), suggesting their suitability for biomedical applications[40-42]. Additionally, a thorough rheological characterization using an oscillatory rheometer defined the gel point as the condition at which the shear storage modulus $\mathrm{G}^{\prime}$ equaled the shear loss modulus G". 

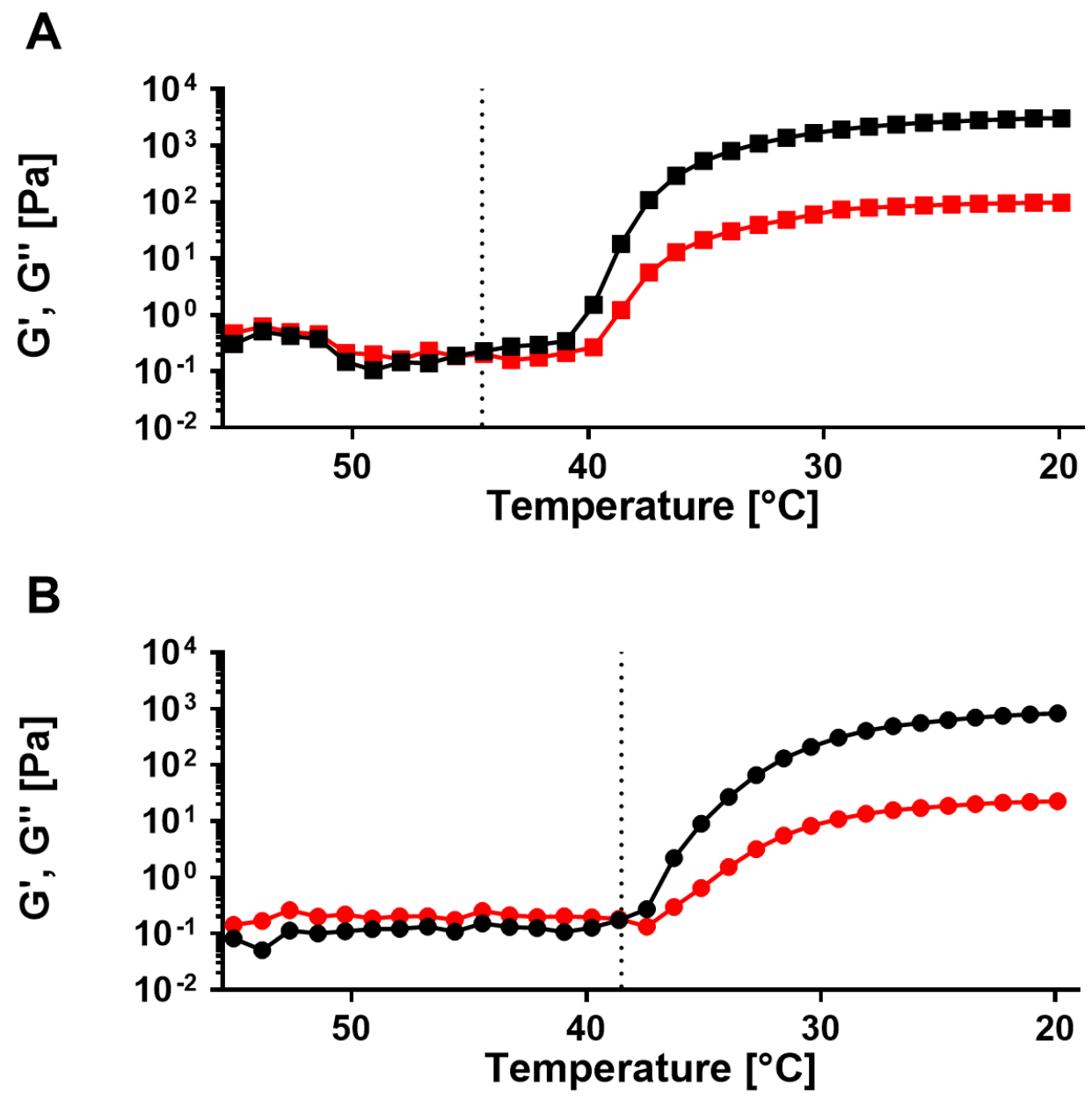

Figure 4. Rheological characterization. Oscillatory test under linear cooling ramp to evaluate the sol-gel transition for (A) cIBU-HG (G' $\boldsymbol{\bullet}$ black, G" $\boldsymbol{\square}$ red) and (B) ref-HG (G' $\bullet$ black, G" $\bullet$ red) hydrogels. Dotted line indicates cross-over point.

Figure 4 shows the rheological behavior of cIBU-HG and ref-HG under a linear cooling ramp from $55^{\circ} \mathrm{C}$ to $20{ }^{\circ} \mathrm{C}$. This temperature range was chosen to observe the material sol-gel transition, considering that the polycondensation begins after the microwave irradiation at $80{ }^{\circ} \mathrm{C}$ and proceeds until room temperature. In ref-HG samples, the gel point is detectable at $38.5^{\circ} \mathrm{C}$, whereas cIBU-HG specimens present the transition state at $44{ }^{\circ} \mathrm{C}$. This difference could be ascribed to the presence of propanediol that eases the nucleation of the early microgel phase, thereby anticipating the sol-to-gel transition of the whole system. 
The progressive generation of local networks and the macromer mobility could be considered responsible of the non-linear trend in the temperature range $55-48{ }^{\circ} \mathrm{C}$. Moreover, in both samples, $\mathrm{G}^{\prime}$ becomes one order of magnitude higher than $\mathrm{G}$ " with a plateau starting at ca. $25^{\circ} \mathrm{C}$, indicating gelation completion. In particular, cIBU-HG are characterized by higher values of storage and loss moduli than ref-HG (cIBU-HG: G' = $3046 \mathrm{~Pa}, \mathrm{G}^{\prime \prime}=97.4 \mathrm{~Pa}$; ref-HG: $\mathrm{G}^{\prime}=830 \mathrm{~Pa}, \mathrm{G}^{\prime \prime}=22.5 \mathrm{~Pa}$ ). This could be explained as a contribution of diol lateral chains of cIBU-HG to the elastic response of the system, and resulting in viscoelastic properties that are in a suitable range for biomedical application[27, 42, 43].

\subsection{IBU release and $\mathrm{COX}$ inhibition effect}

The drug release behavior of the cIBU-HG system was investigated at neutral $\mathrm{pH}$, as well as in acidic and basic environments that are representative of inflammation or diseases[44-46]. Release profiles were compared to that of ref-HG, where IBU is only physically entrapped.

A

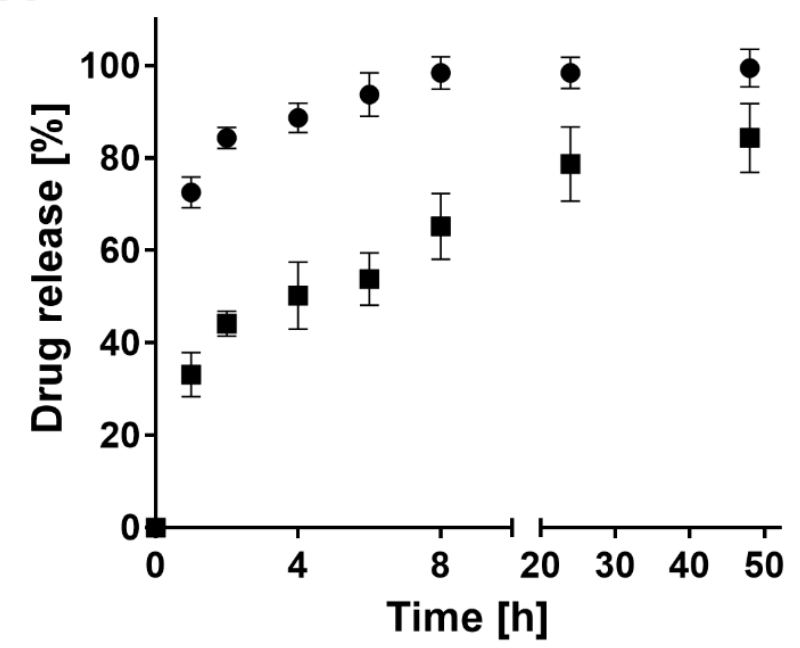

B

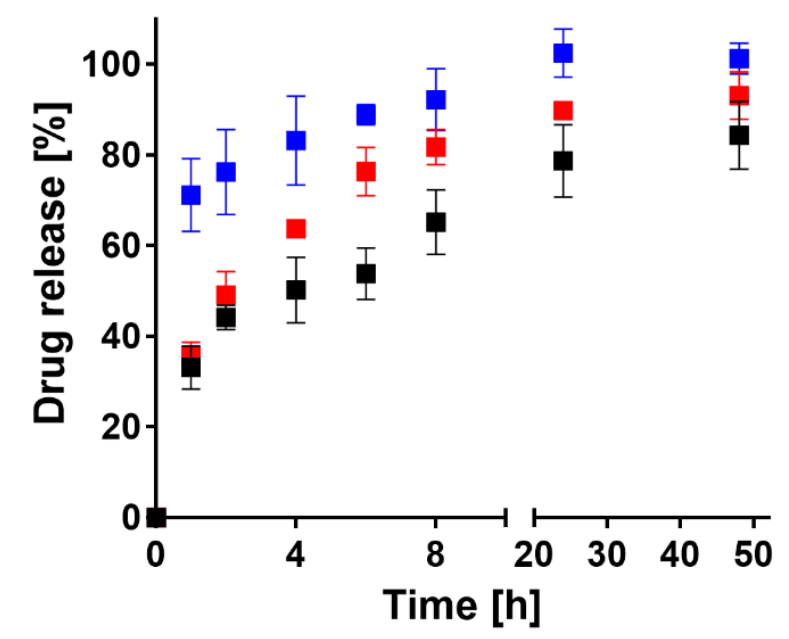

Figure 5. A) Release profiles of IBU from ref-HG (•, black) and cIBU-HG samples ( $\boldsymbol{\bullet}$, black) at pH 7.4; B) Release profiles of IBU from cIBU-HG samples at pH 4.5 ( $\boldsymbol{\square}$, red), $7.4(\boldsymbol{\square}$, black) and 8.5 ( $\boldsymbol{\square}$, blue). 
Figure 5A compares the drug release profiles between cIBU-HG and ref-HG samples at physiological $\mathrm{pH}$ : in ref-HG, an almost complete release of IBU was observed within the first $6 \mathrm{~h}$, whereas cIBU-HG was characterized by a more protracted profile, ensuring sustained IBU release for a longer time (at least $48 \mathrm{~h}$ ). Therefore, the proposed strategy seems to provide a better release performance. Moreover, considering the trends reported in Figure 5B, release from cIBU-HG was pH-sensitive: at acidic or basic $\mathrm{pH}$ levels, we report an increased hydrolytic rate, with the alkaline conditions showing the fastest release. $+10 \%$ cumulative release in acidic medium and $+20 \%$ cumulative release in alkaline medium at $24 \mathrm{~h}$, compared to neutral $\mathrm{pH}$. This means that it is possible to tune the drug release profile according to the $\mathrm{pH}$, demonstrating the potential of this drug delivery system in different biological scenarios. According to the different concentration of $\mathrm{H}^{+}$or $\mathrm{OH}^{-}$, IBU could be released through acid-catalyzed hydrolysis below $\mathrm{pH} 6$, alkaline hydrolysis above $\mathrm{pH} 8$, or neutral hydrolysis when the rate of reaction is independent from the acid-base concentration[33, 47]. In summary, the obtained results demonstrate a reduction in IBU delivery rate, that could be arranged tuning the $\mathrm{pH}$-dependent lability of the linker with a consequent control over the release profile compared to hydrogel systems where the drug is only physically entrapped and no variations in terms of dosage or available amounts are possible in situ.

In addition, even if the hydrogels were formed by polycondensation, which results in an esterification reaction, the high cross-linking density guarantees that hydrolysis first affects the bonds between IBU and the gel network, and only afterwards becomes significant as a degradation mechanism of the polymer backbone. Quantitative analysis of degradation was performed in previous work, showing that the mass degradation occurred at least after 14 days $[48,49]$. Moreover, the physico-chemical behavior of agarose after gelation creates micro-concentrated junction areas, assisted by formation of intramolecular hydrogen bonds that contribute to the stability of the final hydrogel structure[50, 51]. 
Finally, since the chemical modification of IBU with propanediol could potentially affect its therapeutic activity, we investigated in vitro the performance of released drug as a COX inhibitor to rule out possible concerns in terms of reduced biological efficacy. This aspect represents a crucial crossroad to further studies on the biomaterial and to recognize its application to treat inflammation or diseases.

Of the two main COX isoenzymes, COX-1 is normally involved in cellular homeostasis and is constitutively expressed in many tissues, in particular gastric mucosa and kidney, whereas COX-2 presents high expression during inflammatory conditions[52, 53]. The anti-inflammatory activity of IBU was tested by slightly modifying a commercial fluorometric assay for the detection of COX in cell lysates, through the detection of the peroxidase activity, as a result of the formation of prostanoids from arachidonic acid. In our settings, we compared the inhibitory effect of the released IBU to that of ibuprofen sodium salt at the same concentration. The salt form of ibuprofen has a higher solubility in water and, generally, represents the preferred form of the drug, ensuring fast absorption and fast relief, to overcome the limitations related to the presence of the terminal carboxyl moiety. However, the use of ibuprofen sodium salt does not ensure controlled and tunable drug release (Figure 5A) due to the rapid diffusion mechanism related to the hydrophilic molecules in aqueous environment. Figure 6 illustrates the COX inhibitory effect of the investigated samples. 
A

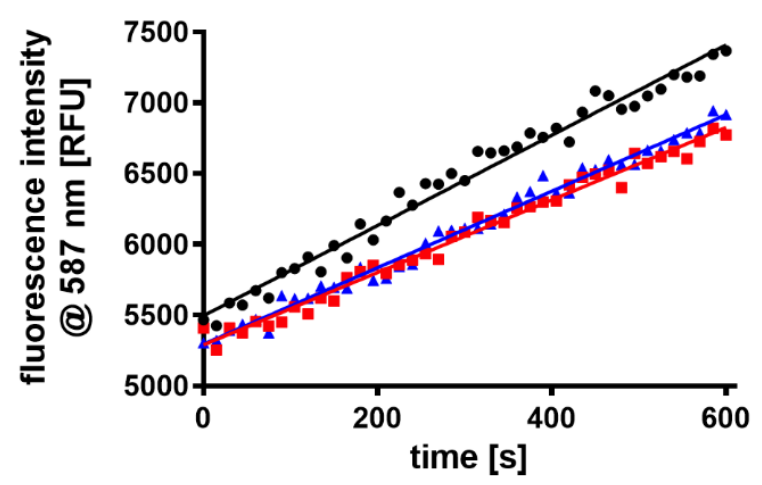

B

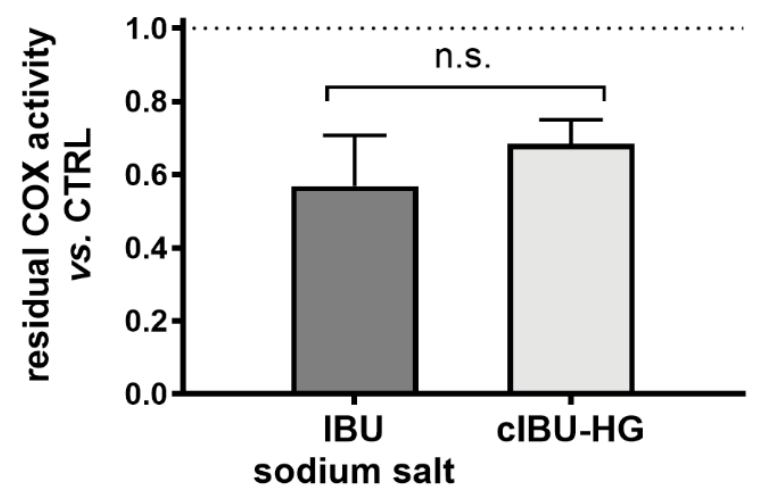

Figure 6. A) COX assay: no inhibitor (CTRL, $\bullet$ black), ibuprofen sodium salt ( $\square$ red) and cIBU-HG eluate blue). B) Residual COX activity after the drug administration: statistical analysis using unpaired t-test; CTRL (dotted line) was set to 1 .

The measurements of COX activity in the absence (hereinafter CTRL) and in the presence of IBU are plotted as relative fluorescence unit (RFU) over time (Figure 6A). A decrease in the slopes was reported for both IBU-containing groups vs. CTRL sample. In particular, no significant differences in terms of COX inhibitory effect have been recorded between IBU sodium salt and cIBU-HG specimens (Figure 6B). This means that the chemical functionalization IBU-propanediol did not alter the antiinflammatory property of the drug and the ester hydrolysis was able to release IBU in its active form. 


\section{Conclusion}

We have designed a functionalized hydrogel for the controlled drug release of IBU, a well-known NSAID, under different $\mathrm{pH}$ conditions, exploiting a cleavable ester bond between the drug in its pristine carboxylic form and the polymeric scaffold. The modification of the terminal IBU carboxyl group does not affect its COX inhibitory activity, ensuring a therapeutic effect that is comparable to that of its salt form. At the same time, the chemical functionalization allows the administration of IBU in the form of free carboxylic acid as a valid alternative to its sodium salt, which is characterized by higher solubility, but at the same time by rapid diffusion that hampers its sustained release. Indeed, our results confirm that the introduction of an IBU-diol derivative in the hydrogel formulation results in a more sustained release profile (8-fold change) than the salt form. Therefore, in light of their physicochemical features, the proposed hydrogel can be considered a promising injectable biomaterial for application in a wide range of inflammatory conditions. 


\section{References}

[1] I. Jake, A. Afrina, I. Nazrul, Formulation and delivery strategies of ibuprofen: challenges and opportunities, Drug Dev Ind Pharm, 44 (2018) 1563-1563.

[2] A. Zarghi, S. Arfaei, Selective COX-2 Inhibitors: A Review of Their Structure-Activity Relationships, Iran J Pharm Res, 10 (2011) 655-683.

[3] B.J. Orlando, M.J. Lucido, M.G. Malkowski, The structure of ibuprofen bound to cyclooxygenase2, J Struct Biol, 189 (2015) 62-66.

[4] R.A. Moore, S. Derry, P.J. Wiffen, S. Straube, D.J. Aldington, Overview review: Comparative efficacy of oral ibuprofen and paracetamol (acetaminophen) across acute and chronic pain conditions, Eur J Pain, 19 (2015) 1213-1223.

[5] P.C.H. Wong, P.W.S. Heng, L.W. Chan, Determination of Solid State Characteristics of SprayCongealed Ibuprofen Solid Lipid Microparticles and Their Impact on Sustaining Drug Release, Mol Pharmaceut, 12 (2015) 1592-1604.

[6] C. Sostres, C.J. Gargallo, A. Lanas, Nonsteroidal anti-inflammatory drugs and upper and lower gastrointestinal mucosal damage, Arthritis Res Ther, 15 (2013).

[7] J.L. Goldstein, B. Cryer, Gastrointestinal injury associated with NSAID use: a case study and review of risk factors and preventative strategies, Drug Healthc Patient, 7 (2015) 31-41.

[8] F. Buttgereit, G.R. Burmester, L.S. Simon, Gastrointestinal toxic side effects of nonsteroidal antiinflammatory drugs and cyclooxygenase-2-specific inhibitors, Am J Med, 110 (2001) 13-19.

[9] K. Stoyanova, Z. Vinarov, S. Tcholakova, Improving Ibuprofen solubility by surfactant-facilitated self-assembly into mixed micelles, J Drug Deliv Sci Tec, 36 (2016) 208-215.

[10] R.P. Dugar, B.Y. Gajera, R.H. Dave, Fusion Method for Solubility and Dissolution Rate Enhancement of Ibuprofen Using Block Copolymer Poloxamer 407, AAPS PharmSciTech, 17 (2016) 1428-1440.

[11] F.E. Koc, M. Senel, Solubility enhancement of Non-Steroidal Anti-Inflammatory Drugs (NSAIDs) using polypolypropylene oxide core PAMAM dendrimers, Int J Pharm, 451 (2013) 18-22.

[12] N.K. Jain, U. Gupta, Application of dendrimer-drug complexation in the enhancement of drug solubility and bioavailability, Expert Opin Drug Met, 4 (2008) 1035-1052.

[13] T. Kitak, A. Dumicik, O. Planinsek, R. Sibanc, S. Srcic, Determination of Solubility Parameters of Ibuprofen and Ibuprofen Lysinate, Molecules, 20 (2015) 21549-21568. 
[14] T.E. Sintra, K. Shimizu, S.P.M. Ventura, S. Shimizu, J.N.C. Lopes, J.A.P. Coutinho, Enhanced dissolution of ibuprofen using ionic liquids as catanionic hydrotropes, Phys Chem Chem Phys, 20 (2018) 2094-2103.

[15] M. Zhao, Y. Huang, Y. Chen, J. Xu, S. Li, X. Guo, PEG-Fmoc-Ibuprofen Conjugate as a Dual Functional Nanomicellar Carrier for Paclitaxel, Bioconjugate Chem, 27 (2016) 2198-2205.

[16] R.A. Moore, S. Derry, S. Straube, J. Ireson-Paine, P.J. Wiffen, Faster, higher, stronger? Evidence for formulation and efficacy for ibuprofen in acute pain, Pain, 155 (2014) 14-21.

[17] M.J. Koenigsknecht, J.R. Baker, B. Wen, A. Frances, H. Zhang, A. Yu, T. Zhao, Y. Tsume, M.P. Pai, B.E. Bleske, X. Zhang, R. Lionberger, A. Lee, G.L. Amidon, W.L. Hasler, D. Sun, In Vivo Dissolution and Systemic Absorption of Immediate Release Ibuprofen in Human Gastrointestinal Tract under Fed and Fasted Conditions, Mol Pharmaceut, 14 (2017) 4295-4304.

[18] R. Francis, D.K. Baby, D.S. Kumar, Poly(N-isopropylacrylamide) hydrogel: Effect of hydrophilicity on controlled release of ibuprofen at different pH, J Appl Polym Sci, 124 (2012) 50795088.

[19] E. Mauri, A. Sacchetti, N. Vicario, L. Peruzzotti-Jametti, F. Rossi, S. Pluchino, Evaluation of RGD functionalization in hybrid hydrogels as 3D neural stem cell culture systems, Biomater Sci, 6 (2018) 501-510.

[20] J. Z. Pan, Y. Jin, S. Q. Lai, L. J. Shi, W. H. Fan, Y. C. Shen, An antibacterial hydrogel with desirable mechanical, self-healing and recyclable properties based on triple-physical crosslinking, Chem Eng, J, 370 (2019), 1228-1238.

[21] M. M. Chen, J. Tian, Y. Liu, H. Cao, R. Y. Li, J. H. Wang, J. L. Wu, Q. Q. Zhang, Dynamic covalent constructed self-healing hydrogel for sequential delivery of antibacterial agent and growth factor in wound healing, Chem Eng J, 373 (2019), 413-424.

[22] X.M. Li, Q.Q. Sun, Q. Li, N. Kawazoe, G.P. Chen, Functional Hydrogels With Tunable Structures and Properties for Tissue Engineering Applications, Front Chem, 6 (2018).

[23] C.A. DeForest, E.A. Sims, K.S. Anseth, Peptide-Functionalized Click Hydrogels with Independently Tunable Mechanics and Chemical Functionality for 3D Cell Culture, Chem Mater, 22 (2010) 4783-4790.

[24] E. Blasco, M.B. Sims, A.S. Goldmann, B.S. Sumerlin, C. Barner-Kowollik, 50th Anniversary Perspective: Polymer Functionalization, Macromolecules, 50 (2017) 5215-5252.

[25] E. Mauri, F. Rossi, A. Sacchetti, Tunable drug delivery using chemoselective functionalization of hydrogels, Mat Sci Eng C, 61 (2016) 851-857. 
[26] F. Rossi, G. Perale, G. Storti, M. Masi, A library of tunable agarose carbomer-based hydrogels for tissue engineering applications: The role of cross-linkers, J Appl Polym Sci, 123 (2012) 2211-2221.

[27] I. Caron, F. Rossi, S. Papa, R. Aloe, M. Sculco, E. Mauri, A. Sacchetti, E. Erba, N. Panini, V. Parazzi, M. Barilani, G. Forloni, G. Perale, L. Lazzari, P. Veglianese, A new three dimensional biomimetic hydrogel to deliver factors secreted by human mesenchymal stem cells in spinal cord injury, Biomaterials, 75 (2016) 135-147.

[28] N. Sun, T. Wang, X.F. Yan, Synthesis and investigation of a self-assembled hydrogel based on hydroxyethyl cellulose and its in vitro ibuprofen drug release characteristics, RSC Adv, 7 (2017) 95009511.

[29] K.T. Campbell, D.Q.M. Craig, T. McNally, Modification of Ibuprofen Drug Release from Poly( ethylene glycol) Layered Silicate Nanocomposites Prepared by Hot- Melt Extrusion, J Appl Polym Sci, 131 (2014).

[30] M. Redpath, C.M.G. Marques, C. Dibden, A. Waddon, R. Lalla, S. MacNeil, Ibuprofen and hydrogel-released ibuprofen in the reduction of inflammation-induced migration in melanoma cells, $\mathrm{Br}$ J Dermatol, 161 (2009) 25-33.

[31] M. Tsintou, K. Dalamagkas, A. Seifalian, Injectable Hydrogel versus Plastically Compressed Collagen Scaffold for Central Nervous System Applications, Int J Biomater, (2018).

[32] J.H. Tian, V.J. Stella, Degradation of Paclitaxel and Related Compounds in Aqueous Solutions III: Degradation Under Acidic pH Conditions and Overall Kinetics, J Pharm Sci, 99 (2010) 1288-1298.

[33] D. Stefanidis, W.P. Jencks, General Base Catalysis of Ester Hydrolysis, J Am Chem Soc, 115 (1993) 6045-6050.

[34] A. Salvi, P.A. Carrupt, J.M. Mayer, B. Testa, Esterase-like activity of human serum albumin toward prodrug esters of nicotinic acid, Drug Metab Dispos, 25 (1997) 395-398.

[35] T. Fukami, T. Yokoi, The Emerging Role of Human Esterases, Drug Metab Pharmacokinet, 27 (2012) 466-477.

[36] P.T. Wong, S.K. Choi, Mechanisms of Drug Release in Nanotherapeutic Delivery Systems, Chem Rev, 115 (2015) 3388-3432.

[37] R. Wolfenden, Y. Yuan, The "Neutral" Hydrolysis of Simple Carboxylic Esters in Water and the Rate Enhancements Produced by Acetylcholinesterase and Other Carboxylic Acid Esterases, J Am Chem Soc, 133 (2011) 13821-13823.

[38] Q. Fu, Y. Wang, Y. Ma, D. Zhang, J.K. Fallon, X. Yang, D. Liu, Z. He, F. Liu, Programmed Hydrolysis in Designing Paclitaxel Prodrug for Nanocarrier Assembly, Sci Rep, 5 (2015). 
[39] S. Ramukutty, E. Ramachandran, Growth, spectral and thermal studies of ibuprofen crystals, Cryst Res Technol, 47 (2012) 31-38.

[40] Y. Li, J. Rodrigues, H. Tomás, Injectable and biodegradable hydrogels: gelation, biodegradation and biomedical applications, Chem Soc Rev, 41 (2012) 2193-2221.

[41] F. Ganji, M.J. Abdekhodaie, A. Ramazani, Gelation time and degradation rate of chitosan-based injectable hydrogel, J Sol-Gel Sci Techn, 42 (2007) 47-53.

[42] A. Sacchetti, E. Mauri, M. Sani, M. Masi, F. Rossi, Microwave-assisted synthesis and click chemistry as simple and efficient strategy for RGD functionalized hydrogels, Tetrahedron Lett, 55 (2014) 6817-6820.

[43] T.T. Xia, W.Q. Liu, L. Yang, A review of gradient stiffness hydrogels used in tissue engineering and regenerative medicine, J Biomed Mater Res A, 105 (2017) 1799-1812.

[44] M.Z.I. Khan, Z. Prebeg, N. Kurjakovic, A pH-dependent colon targeted oral drug delivery system using methacrylic acid copolymers - I. Manipulation of drug release using Eudragit (R) L100-55 and Eudragit (R) S100 combinations, J Control Release, 58 (1999) 215-222.

[45] M.B. Boggara, A. Faraone, R. Krishnamoorti, Effect of $\mathrm{pH}$ and Ibuprofen on the Phospholipid Bilayer Bending Modulus, J Phys Chem B, 114 (2010) 8061-8066.

[46] H. Matsui, O. Shimokawa, T. Kaneko, Y. Nagano, K. Rai, L. Hyodo, The pathophysiology of nonsteroidal anti-inflammatory drug (NSAID)-induced mucosal injuries in stomach and small intestine, $\mathrm{J}$ Clin Biochem Nutr, 48 (2011) 107-111.

[47] C. Ding, Z. Li, A review of drug release mechanisms from nanocarrier systems, Mat Sci Eng C, 76 (2017) 1440-1453.

[48] E. Mauri, E. Micotti, A. Rossetti, L. Melone, S. Papa, G. Azzolini, S. Rimondo, P. Veglianese, C. Punta, F. Rossi, A. Sacchetti, Microwave-assisted synthesis of TEMPO-labeled hydrogels traceable with MRI, Soft Matter, 14 (2018) 558-565.

[49] F. Rossi, X. Chatzistavrou, G. Perale, A.R. Boccaccini, Synthesis and degradation of agarcarbomer based hydrogels for tissue engineering applications, J Appl Polym Sci, 123 (2012) 398-408.

[50] E. Fernández, D. López, C. Mijangos, M. Duskova-Smrckova, M. Ilavsky, K. Dusek, Rheological and thermal properties of agarose aqueous solutions and hydrogels, J Polym Sci Pol Phys, 46 (2008) 322-328.

[51] B. Mao, T. Divoux, P. Snabre, Normal force controlled rheology applied to agar gelation, J Rheol, 60 (2016) 473-489. 
[52] W.L. Xie, J.G. Chipman, D.L. Robertson, R.L. Erikson, D.L. Simmons, Expression of a MitogenResponsive Gene Encoding Prostaglandin Synthase Is Regulated by Messenger-Rna Splicing, Proc Natl Acad Sci USA, 88 (1991) 2692-2696.

[53] I. Morita, Distinct functions of COX-1 and COX-2, Prostag Oth Lipid M, 68-9 (2002) 165-175. 